\title{
Scanning mechanism based on a programmable liquid crystal display
}

\author{
M.G. Capeluto, C. La Mela, C. Iemmi, M.C. Marconi* \\ Departamento de Física, Facultad de Ciencias Exactas y Naturales, Universidad de Buenos Aires, C1428EHA Buenos Aires, Argentina
}

Received 20 October 2003; received in revised form 9 December 2003; accepted 30 December 2003

\begin{abstract}
In this work we propose a new robust optoelectronic scanning system for scanning microscopy. The device is based on a programmable liquid crystal television display (LCTV) that acts as a pixel-by-pixel controlled pure phase spatial light modulator. The computer addressed liquid crystal screen allows synthesize different diffractive optical elements at video rates. The phase shifts introduced by these elements make possible a complete control over the position and focusing of the scanning beam.
\end{abstract}

(C) 2004 Published by Elsevier B.V.

Keywords: Scanning microscopy; Liquid crystal TV; Optical diffractive elements; Digital image processing; Scanners

\section{Introduction}

Imaging of fluorescent samples by scanning microscopy has produced an important impact in the development of new diagnostic techniques in biology, medicine and other sciences. One of the main difficulties that appear when images of biological specimens are acquired is the low speed of typical scanning systems. To improve the performance of scanning microscopes, many efforts have been made to design new, fast and reliable scanning mechanisms. Fast sweep of the excitation beam over the sample [1] was achieved using a

\footnotetext{
${ }^{*}$ Corresponding author. Tel.: +54-11-4576-3300x346; fax: +54-11-4576-3357.

E-mail address: marconi@df.uba.ar (M.C. Marconi).
}

high-speed rotating polygonal mirror combined with a CCD camera. The spatial information in this system is encoded by the timing of the raster scan pattern as in typical confocal microscopy. The scan system uses a galvanometer mirror combined with a fast rotating polygonal mirror to rapidly raster scan the illuminating laser beam across a sample plane.

Another option, the bilateral scanning technique [2], is based on a line focus scanning. Scanning a linear focus obtained with a cylindrical lens reduces the image acquisition time by illuminating the image plane with a line instead of a point. The resulting fluorescent line image is subsequently acquired with a spatially resolved detector such as CCD camera.

Multi-focal-point illumination [3] allows multiphoton multi-focal microscopy and is analogous to 
Nipkow disk-based confocal systems. This approach is based on a custom-fabricated scan lens composed of a specially designed lenslet array that focuses the incident laser into a multiple focus spots at the field aperture plane. The microlenses are arranged in a helical pattern so that fast rotation of the disk ensures complete, uniform and fast coverage of the focal plane. Multiple focal-spot scanning also has the advantage of being extremely robust. Another approach is to use resonant galvanometers to drive scanning mirrors [4] in both directions in the sample plane for video rate point detection. The confocal microscope incorporates a resonant galvanometer for horizontal scanning. Compared with the line excitation mode and rotation microlens array approaches, which provide very fast scan rates, this implementation does not require spatial discrimination between the fluorescent photons leading to an increased collection efficiency. Point scanning also means that multiple emission channels can be simultaneously and cheaply collected with perfect registration.

In this work we present a fast and robust scanning mechanism based on a programmable spatial light modulator (SLM). The SLM, consists in a liquid crystal television display, with VGA resolution $(640 \times 480$ pixels $)$, that is configured to work as a pure phase modulator [5]. Working in this mode this device allows to synthesize different diffractive optical elements at video rate and consequently to control the beam position in a optical system by means of a mechanism with no movable parts and specially attractive for scanning microscopy. A unique characteristic of this scanning mechanism compared with previous systems are that it allows to make selected scanning patterns in the object plane to selectively excite determined regions of the sample by programming in the LCD a sequence of different diffractive optical elements. Another unique capability is that also allows axial scanning for a complete 3D scan without the necessity to displace the specimen. In this paper we review the main idea of this new scanning system and discuss the advantages and limitations of the method.

In Section 2 we describe the proposed method and the scanning device. We analyze the ranges that can be reached according to the SLM modulation responses and optical set up. Experimental results are presented in Section 3. Finally in Section 4 are summarized the conclusions.

\section{Description of the scanning device}

Twisted nematic liquid crystals displays (LCDs) have been widely used as spatial light modulators. This kind of modulators consist of a very thin liquid crystal layer sandwiched between two parallel glass plates on which transparent electrodes are patterned. The elongated liquid crystal molecules are aligned along the polishing direction of a layer coated onto the electrodes in each one of the covering glass plates and are normally arranged with a twist angle determined by the relative orientation of the two plates. The twist angle from one surface to the other is in general $90^{\circ}$. This twisted structure generates a macroscopic optical anisotropy in the LCD. The orientation of liquid crystal molecules can be modified by applying an external electric field that changes the anisotropy of the material as a bulk producing a birefringence that can be controlled by changing the applied external field. For most common applications these devices are used between crossed linear polarizers to obtain in this way intensity modulation.

In general, LCDs produce coupled amplitude and phase modulation as a function of the applied voltage. This coupling effect deteriorates the response of ideal optical elements that are designed as phase-only or amplitude-only functions. However, recently it was shown that it is possible to obtain phase-only or amplitude-only modulation by using elliptical polarization configurations [5].

In recent years the manufacture of LCDs has evolved into the production of devices with a large number of pixels that can be addressed individually. In this way it is possible to control the phase or the amplitude of a whole wavefront that impinges the modulator by controlling the phase or the amplitude of each small portion of the beam passing through each LCD pixel. Moreover this task can be performed at video rate. The use of SLMs to implement diffractive optical elements has been reported to be a very useful tool in real time optical processing [6-9]. 
A LCTV placed between a set of properly oriented polarizers and wave plates, as shown schematically in Fig. 1, can act as a programmable phase-only modulator. By programming the phase shifts introduced onto the illumination beam pixel by pixel it is possible to generate a phase shift modulation in the whole wavefront. Programming different spatial phase functions it can be obtained a total phase shift similar to that introduced by a spherical lens, a cylindrical lens or a wedge. For example, in our proposed scanning set up a dynamically changed focal length spherical lens would produce an axial scan of the focal point, or similarly a variable linear phase (wedge) produces a lateral scan in any transversal direction. Also a scan over a complete line can be obtained combining simultaneously a cylindrical lens and a linear wedge. The possibility of programming several optical elements individually or simultaneously is a characteristic unique of this scanning device and makes it extremely versatile. Other options like multi-focal-point illumination are also possible. As the different optical diffractive elements can be refreshed at video rate a fast and robust sweep mechanism totally programmable and immune to mechanical noise can be implemented.

In Fig. 2 a scheme of the scanning system, based on a liquid crystal television display, is shown. As

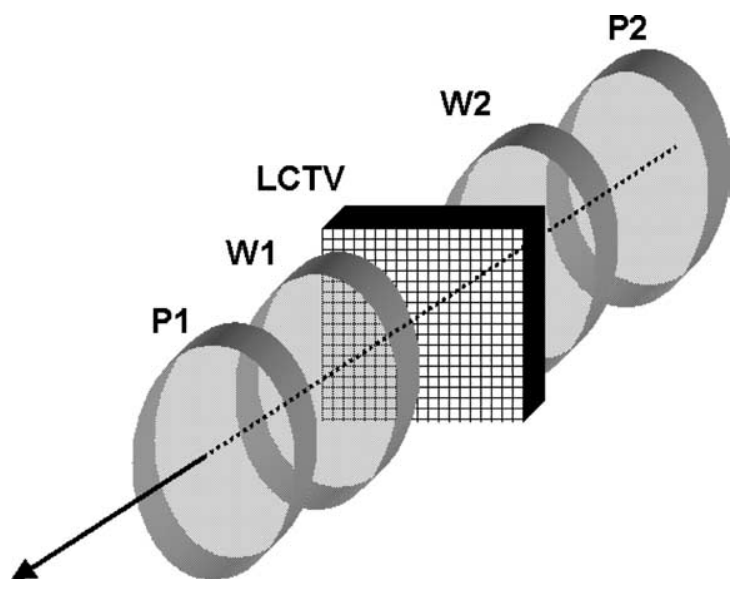

Fig. 1. A LCTV placed between a set of properly oriented polarizers $(\mathrm{P} 1, \mathrm{P} 2)$ and wave plates $(\mathrm{W} 1, \mathrm{~W} 2)$, can act as a programmable phase-only modulator or amplitude-only modulator.

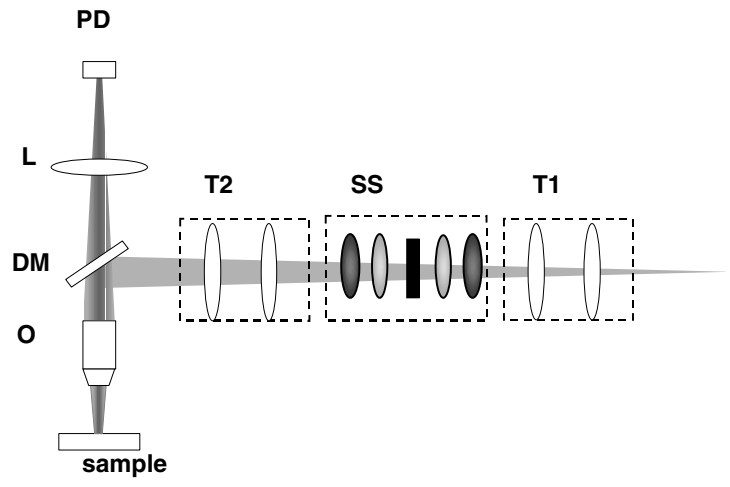

Fig. 2. Scheme of the scanning system placed in the path of the illumination laser beam of a microscope. T1 and T2 are telescopes to adapt the size of the illuminating beam. DM: dichroic mirror. SS: scanning system. O: objective. L: lens. PD: photodetector.

we previously mentioned the phase-only spatial light modulator is conformed by the LCTV between polarizers $\mathrm{P} 1$ and $\mathrm{P} 2$ and waveplates $\mathrm{W} 1$ and W2. The whole set is placed in the path of the illumination laser beam of a microscope. A first telescope $\mathrm{T} 1$ adapts the beam size to fully cover the LCD surface. The number of illuminated pixels will determine the range of the focal distance of the lenses that can be programmed, as will be discussed later. The waveplates and polarizers are oriented with their axis to maintain constant the total transmission of the set and allowing only phase modulation as described in [5]. A second telescope T2 is placed in the beam path to fully coverage the objective aperture allowing for a minimum illumination spot size. The dichroic mirror DM relays the illuminating beam towards the objective while allowing by transmission to gather in a photodetector the fluorescence signal produced.

The LCD was characterized following the procedure mentioned in [5]. Using an $\mathrm{Ar}^{+}$ion laser emitting in a single line at $457 \mathrm{~nm}$, the relative orientation of the polarizers and waveplates was adjusted in such a way to maximize the total transmission of the set and simultaneously producing the maximum phase modulation when the voltage in the electrodes is changed. In Fig. 3 the phase shift and intensity modulation introduced by the LCD as a function of the programmed gray 


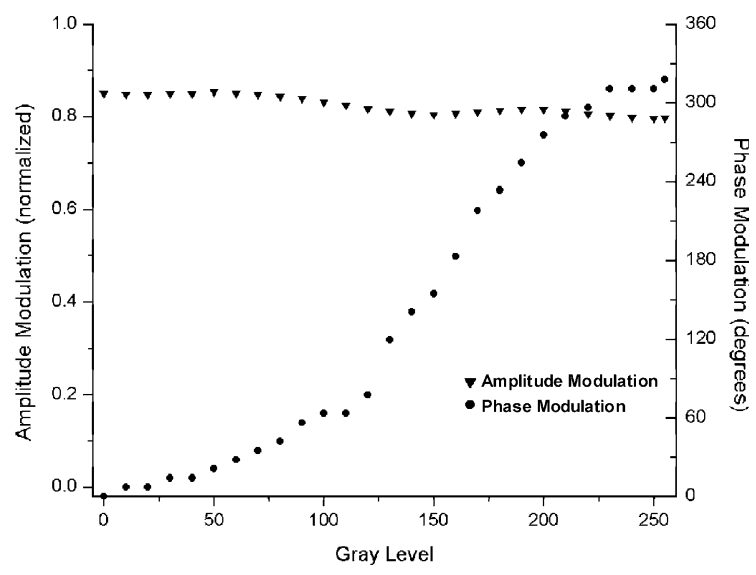

Fig. 3. Phase shift and intensity modulation introduced by the LCD as a function of the programmed gray level.

level is shown. The phase modulation achieved is approximately $330^{\circ}$ while the total amplitude remains almost constant with modulation less than $5 \%$. In order to display a desired phase distribution we generate a calibration phase vs. gray level (applied voltage) look-up table using the modulation curve.

The scanning range attainable with this kind of system strongly depends on the phase depth modulation achievable with the SLM and the LCTV spatial resolution. In our case we used a panel Sony LCX012BL removed from a Proxima 5100 video-projector. This panel has VGA resolution $(640 \times 480$ pixels $)$ with square pixels $34 \mu \mathrm{m}$ and separated by a distance of $41.3 \mu \mathrm{m}$ from center to center.

The scanning system is composed by the SLM and the objective of the microscope. With the SLM different diffractive elements are generated producing the scan, and the microscope objective de-magnify this scan in the sample plane. We will particularly discuss in detail the case of pointscanning illumination. This kind of illumination can be achieved programming the SLM as a variable focal length lens to produce an axial scan or a variable wedge to produce a lateral scan. The phase optical elements are programmed changing the gray level pixel-by-pixel according the curve depicted in Fig. 3.

The maximum phase depth value imposes the condition that the functions that can be displayed onto the SLM must be $2 \pi$ modulus functions. In particular lenses generated with this device belong to the category of diffractive Fresnel lenses. Some authors [6,7] have synthesized phase encoded Fresnel lenses onto a programmable SLM, nevertheless the sampling of the original function by the pixelated structure of the SLM causes the appearance of multiple lenses when the sampling rate is lower than the Nyquist frequency. This effect leads to the presence of secondary maxima in the focal plane with the consequent loss of efficiency in the principal focus. In our case lenses with focal length of about $1 \mathrm{~m}$ and radius of 300 pixels avoid this inconvenient. Taking this into account we selected the distance between the LCD and the principal plane of the objective lens to be $1 \mathrm{~m}$. Utilizing a SL APPO M-Plan $20 \times$ objective with a focal length of $10 \mathrm{~mm}$ and the SLM screen, we calculated that programming lenses with focal distances between $(-\infty,-100 \mathrm{~cm})$ and $(+\infty,+100$ $\mathrm{cm}$ ) it was possible to produce a longitudinal scan with a total depth of $200 \mu \mathrm{m}$. We estimated that for this objective the focal depth is approximately $3.5 \mu \mathrm{m}$. In order to assure the illumination in different planes, the programmed focal lengths were selected to produce changes of $3.5 \mu \mathrm{m}$ in the focal plane location. The axial resolution is determined both by the objective numerical aperture and the modulation capability in the focal distance of the diffractive elements. For example the same scan device combined with $100 \times$ objective would produce an axial resolution of $\sim 1 \mu \mathrm{m}$ changing the focal length of the programmed lens in $\sim 5 \mathrm{~mm}$ (for $f \sim 1 \mathrm{~m})$ but with decreased scan depth $(\sim 30 \mu \mathrm{m})$. Fig. 4 shows the results of the calculation. The capability to program selected values of the focal distance in the SLM avoids the problem of the definition of the pixel size, usual when using continuous scan illumination.

Similarly, the lateral scan is performed programming a variable angle wedge. The $2 \pi$ modulus linear phase function that generates the wedge is equivalent to a blazed diffraction grating. Varying the angle of the wedge (or equivalently the pitch of the diffraction grating) the deflection angle can be changed producing a lateral beam displacement. Using the same distances from the SLM to the objective, and the same considerations for the spot 


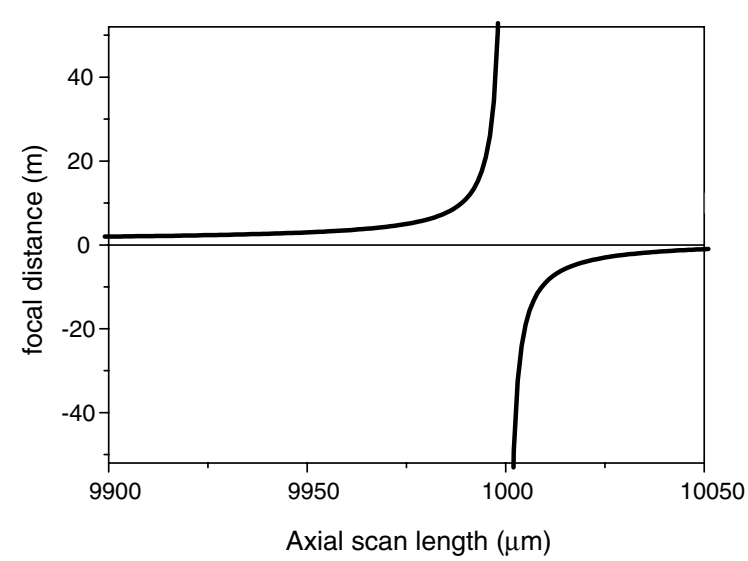

Fig. 4. Calculated values of programmed focal lengths on the LCTV to produce a $200 \mu \mathrm{m}$ scan in axial direction over the sample.

size, we calculated the diffraction gratings periods necessary to obtain a lateral displacement in the focal plane of $1 \mu \mathrm{m}$. Fig. 5 shows the calculated values. These values were calculated taking into account the available periods that can be programmed with our LCTV screen, and the fact that the minimum change in the grating period is \pm 1 pixel, this is $\pm 41.3 \mu \mathrm{m}$. Also the LCD characteristic imposes $6 \mathrm{~mm}^{-1}$ the maximum pitch that can be programmed. With these restrictions the amplitude of the scan for this particular configuration

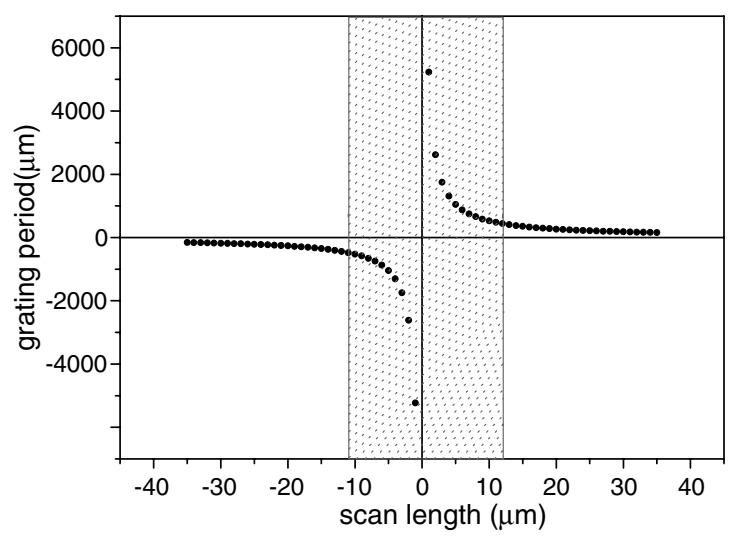

Fig. 5. Calculated values of programmed gratings periods on the LCTV to obtain a scan of $22 \mu \mathrm{m}$ over the sample. The shaded region in the figure is accessible with our experimental set up. is approximately $22 \mu \mathrm{m}$, and can be obtained by programming in the SLM gratings with periods indicated in Fig. 5.

\section{Experimental results}

We tested the capabilities of the scanning system using the experimental set up depicted in Fig. 6. The SLM was illuminated by an Ar laser with $10 \mathrm{~mW}$ tuned in the $457 \mathrm{~nm}$ line. The modulator was connected to a personal computer controlling the video signal to synthesize the different diffractive optical elements. The optical system is completed with an objective lens that produces an image onto the CCD detector with $510 \times 492$ pixels. We demonstrate the capability to produce arbitrary scans in the sample plane (xy) by programming a sequence of wedges in the $x$ direction and combining this scan with a sweep in the $y$ direction. As result, we were able to produce an arbitrary scan that for demonstration purpose has a $Z$ shape, shown in Fig. 7(a). The total span of the programmed scan is 255 pixels $(2.8 \mathrm{~mm})$ in the horizontal direction. However this size can be reduced for different objectives. This value corresponds to the optical system implemented for demonstration purpose. The scan is composed of 61 points (frames), each one generated by a different wedge programmed in the SLM. The complete figure can be scanned in $2 \mathrm{~s}$ refreshing the optical diffractive elements at video rate.

Another example of the capability of this device was demonstrated programming a cylindrical lens, to produce a line-illumination of the sample. Fig. 7(b) shows the result of this test, where the illumination spot is converted in a line focus 2.4

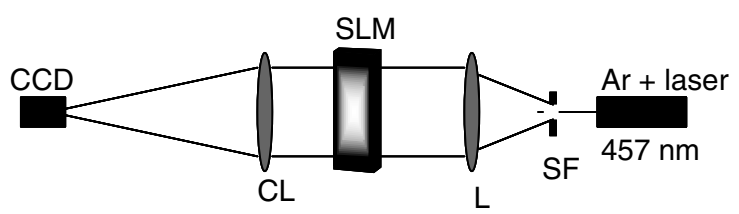

Fig. 6. Experimental set up used to test the capabilities of the scanning system. SF: spatial filter. LC: collimator lens. SLM: spatial light modulator. L: lens. 


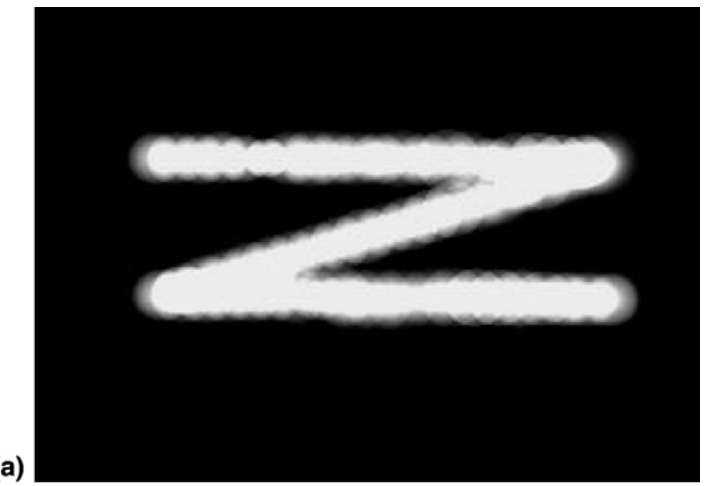

(b)

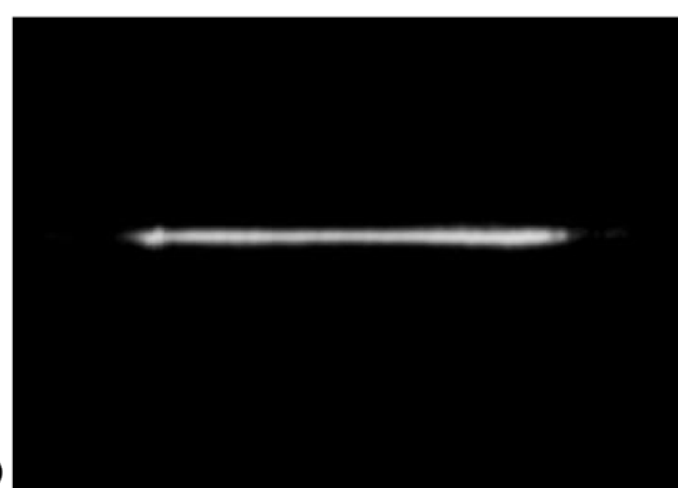

Fig. 7. Arbitrary scanning patterns obtained to test the capabilities of the device. (a) Scan with a $\mathrm{Z}$ shape produced by programming two wedges in the two transverse directions over the sample. The scan size in the horizontal direction is $2.8 \mathrm{~mm}$. (b) Line-illumination of the sample produced by programming a cylindrical lens on the LCTV. The size of the horizontal focal line is $2.4 \mathrm{~mm}$.

$\mathrm{mm}$ long. Other illumination patterns are also possible by the adequate programming of the SLM. Longitudinal scans can be performed by programming a sequence of spherical lenses each one with different focal length in the SLM, in a similar way as described in [9].

\section{Conclusions}

A novel scanning device suitable for scanning microscopy based on the utilization of a SLM that reproduces different diffractive optics elements was presented. The modulator can be programmed at video rate to dynamically change the optical configuration of the focusing optical system of the illuminating laser and produce arbitrary scanning patterns. This capability was demonstrated using a SONY LCX012BL screen. This device can produce accurate arbitrary scanning patterns without any movable part. A unique characteristic of this system is that allows for axial scans by programming spherical lenses of variable powers.

The possibility to superimpose different optical elements simultaneously in the SLM makes this scanning device extremely versatile and as is composed by no movable parts is very stable and robust.

However, the pixeled structure of the screen produces a diffraction of the input beam representing a loss that diminishes the total transmission of the device to less than $10 \%$. Also this pixeled structure limits the optical elements that can be displayed as was discussed before. This limitation can be overcome, if necessary, by the fabrication of custom-made LC screens.

The different diffractive elements that produce illumination in one pixel in the sample can be generated at video rate. Besides this is a limitation to the overall performance of the scan system to produce truly "video rate" image acquisition, the versatility of the device to produce determined scan paths and the robustness of the system are unique advantages.

\section{Acknowledgements}

This work was supported by grants from Buenos Aires University (X216 and TX176) and a grant from ANPCyT (grant PICT 6303). MGC has a fellowship from CONICET. CLM has a fellowship from UBA. CI and MCM are members of the research staff of CONICET.

\section{References}

[1] K.H. Kim, C. Buehler, P.T.C. So, Appl. Opt. 38 (1999) 6004.

[2] G.J. Brakenhoff, J. Squier, T. Norris, A.C. Blinton, M.H. Wade, B. Athey, J. Microsc. 181 (1996) 253.

[3] M. Straub, S. Hell, Bioimaging 6 (1998) 177. 
[4] G.Y. Fan, H. Fujisaki, A. Miyawaki, R.-K. Tsay, R.Y. Tsien, M.H. Ellisman, Biophys. J. 76 (1999) 2412.

[5] A. Marquez, C. Iemmi, I. Moreno, J. Davis, J. Campos, M. Yzuel, Opt. Eng. 40 (2001) 2558.

[6] J. Davis, D. Cottrell, R. Lilly, S. Connely, Opt. Lett. 14 (1989) 420.
[7] D. Cottrell, J. Davis, T. Hedman, R. Lilly, Appl. Opt. 29 (1990) 2505.

[8] J. Davis, J. Escalera, J. Campos, M. Yzuel, C. Iemmi, Opt. Lett. 24 (1999) 628.

[9] A. Marquez, C. Iemmi, J. Escalera, J. Campos, S. Ledesma, J. Davis, M. Yzuel, Appl. Opt. 40 (2001) 2316. 